\title{
GMR \\ Correlation among traits as criterion of cotton genotypes indirect selection
}

\author{
MC Reis², DBO Cardoso', EG Silva Júnior², BC Gomes², LTG Pereira², DA Gomes² and LB Sousa ${ }^{2}$. \\ ${ }^{1}$ Programa de Pós-Graduação em Agronomia, Instituto de Ciências Agrárias, Universidade \\ Federal de Uberlândia, Uberlândia, MG, Brasil \\ ${ }^{2}$ Instituto de Ciências Agrárias, Universidade Federal de Uberlândia, Uberlândia, MG, \\ Brasil \\ Corresponding author: Daniel Bonifácio Oliveira Cardoso \\ E-mail: danieludia13@hotmail.com
}

Genet. Mol. Res. 16 (3): gmr16039805

Received September 2, 2017

Accepted September 28, 2017

Published October 5, 2017

DOI http://dx.doi.org/10.4238/gmr16039805

Copyright @ 2017 The Authors. This is an open-access article distributed under the terms of the Creative Commons Attribution ShareAlike (CC BY-SA) 4.0 License.

\begin{abstract}
The cotton crop has notable importance for Brazilian and worldwide economy. Relationships knowledge among characters allows developing alternatives that increase the genetic progress with simultaneous selection of several traits. The use of correlation estimates for use in indirect selection helps the breeder in the selection of characters and facilitates progress in the selection. This work evaluates the phenotypic correlation and unfolding of the direct and indirect effects of morphological and technological fiber traits as criterion of indirect selection in cotton genotypes. The experiment was carried out at Fazenda Capim Branco, a research station of Universidade Federal de Uberlândia, 2014/2015 season. 21 genotypes were evaluated, 19 commercial genotypes F3 proceeding from the Programa de Melhoramento Genético Algodoeiro (PROMALG) and two commercial control: FM 966 and DP 555. The experimental design was the randomized complete block with four replications. The experimental parcel was made of four rows of 5 meters cotton plants length, spaced of 0.9 meters. The morphological and agronomic traits evaluated were: a) chlorophyll content (TCA and TCB); b) number of fruiting branches (NFB); c) 1st fruit node height $(\mathrm{NH})$; d) first, second and third-position bolls percentage (FPB, SPB, TPB); e) leaf Area Index (LAI); f) cotton seed yield (CSP); g) lint yield (LP); and h) Lint yield (LY). The fiber quality traits evaluated with HVI (High Instrument Volume): i) Fiber Length average (UHML); ii) Uniformity of length (UI); iii) Short fiber Index (SFI); iv) Fiber Strengh (STR); v) Elongation (ELG); vi) Micronaire (MIC), and vii) Fiber Maturity (MR). Statistical analyses and multicollinearity diagnosis were carried out with the software GENES. The intrinsic traits of fiber quality, according to correlations, influenced cotton seed yield and also morphological traits. Based on phenotypic correlations and according to path analysis, the higher micronaire index of favorable effect on cotton seed yield was identified due to a positive correlation and direct effect. Thus, micronaire index can be an indirect selection criterion of cotton genotypes.
\end{abstract}


Keywords: Gossypium Hirsutum, Indirect Selection, Cotton Seed Yield.

\section{INTRODUCTION}

The cotton plant (Gossypium hirsutum L.) is a specie of genus Gossypium, belonging to the family Malvaceae (CARDOSO et al., 2015). Cotton fiber is the main product, which is the most natural fiber used through textile industry in the world (CONAB, 2015).

Brazil is the fifth largest cotton producer throughout world, behind India, China, USA and Pakistan. It is the third largest exporter of cotton fiber, in addition, has the highest upland cotton grown suitable for rainfed agriculture (ABRAPA, 2017).

The cotton plantion area in the country was 938.2 thousand hectares in 2016/2017 season, and the projected production of cotton is 3.72 million tons. The decrease in planted area was $1.7 \%$; however, the production increased $15 \%$ compared to the previous season. It happens, specially, because of edaphoclimatic conditions favorable to culture and advances in genetic breeding (CONAB, 2017).

The textile industry search for genotypes with high yield and fiber quality, which is a challenge to cotton genetic breeding identify superior genotypes, mainly with complex traits, such as yield, because they are controlled by many genes, that is, a polygenic traits (RIOS et al., 2012; ENTRINGER et al., 2014).

The use of correlation estimates for use in indirect selection helps the breeder in the selection of characters and facilitates progress in the selection (RIOS et al., 2012). Correlation is a statistical parameter that measures the degree of relationship among traits, which may be negative or positive (RAMALHO et al., 2012). The association among traits is evaluated through phenotypic, genotypic and environmental correlations. However, only phenotypic and genotypic correlations involve association of inheritable nature, and so, they are of greater importance to guide a plant-breeding program (AZEVEDO et al., 2016). However, the correlation evaluated may not represent the true association between two traits, since high or low correlation may occur influenced by a third or set of traits (TOEBE; CARGNELUTTI SON, 2013).

Path analysis determines the influence among traits and provides the most detailed understanding of the relationships among them by estimating coefficients that define the influence of one trait action in another. Thus, the correlation between two traits can be deployed in direct and indirect effects, can identify type and degree of correlation between them, as well as, provide important and useful information to the breeder during the selection process (TEIXEIRA et al., 2012; LÚCIO et al., 2013).

Concomitant with the use of path analysis should have multicollinearity diagnosis of data to avoid systematic error in results, which distorts evaluation of direct and indirect effects. Therefore, two basic strategies for path analysis are used: identification and elimination of traits responsible for collinearity; or path and ridge regression analysis, when the researcher chooses do not eliminate the variables (SALLA et al., 2015).

This study evaluates the phenotypic correlation and the unfolding of direct and indirect effects of the morphological and technological fiber traits as cotton genotypes selection criterion.

\section{MATERIALS AND METHODS}

The experiment was carried out at Fazenda Capim Branco, a research station of Universidade Federal de Uberlândia, between the geographical coordenates $18^{\circ} 52^{\prime} \mathrm{S} ; 48^{\circ} 20^{\prime} \mathrm{W}$ and $805 \mathrm{~m}$ of altitude, in Uberlândia, Minas Gerais, during 2014/2015.

The soil characterization is Latossolo Vermelho Escuro Distrófico (Brazilian classification system). Before starting the experiment, the soil sample was made, after that, composite soil sampling was collected, sent to the laboratory for chemical and physical analysis of the soil for the purpose of liming and fertilization. Soil preparation was done using a conventional tillage, with one plowing and two harrowings. The area was grooved with row spacing of ninety centimeters before sowing, then, fertilization and soil acidity correction were made according to crop's need.

21 genotypes of white fiber cotton were evaluated and 19 were genotypes F3 proceeding from Programa de Melhoramento Genético Algodoeiro (PROMALG): UFUJP15 - A, UFUJP15 - B, UFUJP15 - C, UFUJP15 


\begin{abstract}
- D, UFUJP15 - E, UFUJP15 - F, UFUJP15 - G, UFUJP15 - H, UFUJP15 - I, UFUJP15 - J, UFUJP15 K, UFUJP15 - L, UFUJP15 - M, UFUJP15 - N, UFUJP15 - OB, UFUJP15 - P, UFUJP15 - S, UFUJP15 T, UFUJP15 - Z, and two commercial cultivars: FM 966 (Fibermax) and DP 555 (Deltapine). The experimental design was the randomized complete block (RCB) with three replications. The plots consisted of four rows of 5 meters long with row spacing of 0.9 meters. The plot floor area are the two central rows, discarding the soybean plants to $0.5 \mathrm{~m}$ from the borders of each row.
\end{abstract}

The seed rate was sixteen seeds per linear meter planted $2 \mathrm{~cm}$ depth. The thinning occurred thirty days after seedling emergence, with the goal to establish a plant density of 8 plants per linear meter, adopting the population density of 88.890 plants per hectare (ha-1). Before the sowing, the seeds were treated with a Fipronil insecticide, commercially available as Standak [01] ® [02] and the systemic fungicide formulation Carboxine with a contact fungicide, Tiram, commercially available as Vitvax ${ }^{\circledR}$ Thiram $200 \mathrm{SC}$, both were used at doses of $450 \mathrm{~mL}$ of the commercial product for every $100 \mathrm{~kg}$ of seeds.

During the cotton cycle several treatments were made, among them the control of weeds, with the application of pre-emergency action herbicide, commercial product Dual Gold at the dose of 1.5 liters per hectare. In postemergence were used commercial products Gliover and Gramoxone 200, both at a dose of $1.5 \mathrm{~L}$ ha-1 and the applications were made in directed jet, supplemented with manual weeding, when necessary. The occurrence of diseases as - Black Spot (Alternaria sp.) and Ramularia (Ramularia areola); insect pests with the incidence of Tripes (Frankliniella schultzei), Melon or Cotton Aphid (Aphis gossypii), Cotton leafworm (Alabama argillacea), Boll Weevil (Anthonomus grandis)), Cotton Stainer (Dysdercus spp.) - caused periodic sampling, and for the control were made applications of phytosanitary products recommended for the crop. The growth bioregulator, with commercial name of Pix ${ }^{\circledR} \mathrm{HC}$, was used for the vegetative growth control of cotton plants. Boric acid applications in the full flowering stage ( 80 to $90 \mathrm{DAE}$ ) were made with the purpose of conditioning higher flowering set and good development of bolls.

Five plants were randomly selected in each plot, so during plants development and harvest stages, morphological and agronomic evaluations were done: a) Chlorophyll content (TCA and TCB) - determined in two leaves of middle third of each plant with the Electronic Chlorophyll Content Meter (CFL 1030); b) Number of fruiting branches (NFB) - visual quantification of fruiting branches in each plant, the fruiting branch presents sympodial growth traits and zig-zag shape; c) 1st fruit node height $(\mathrm{NH}, \mathrm{cm})$ - the distance in centimeters between soil surface and first fruit node insertion in each plant; d) Number of first-position boll (FPB); e) Number of secondposition boll (SPB); f) Number of third position boll (TPB) - visual quantification of boll's total number in each single position, which were later determined in boll percentage of each position according to evaluation in each plant of plot floor area; g) Leaf area index (LAI, cm) - consider the middle third of two leaves in each plant, measure the central vein length of each leaf in centimeters with graduated ruler. The leaf area is calculated through average values of central vein length according to Fideles Filho et al (2010) equation: Y= 0.7254 (x)2.08922, where $\mathrm{Y}$ is the result of leaf area and $\mathrm{X}$ is the leaf's central vein length; $\mathrm{h}$ ) Cotton seed yield (CSP, kg ha-1) - the picked cotton lints of each plot floor area were later weighed in a digital scale, and from this weight and square area of plot floor area were evaluated the yield in kilograms of cotton seed per hectare; i) Lint yield (kg ha-1) (LP) - fiber weight after processing of cotton seed lint, and this yield weight was in kilograms per hectare; j) Lint yield (\%) LY - fiber weight divided by cotton seed weight, and this value is multiplied by 100 to obtain the value of lint yield in percentage.

The fiber quality traits were evaluated with HVI (High Instrument Volume) that consider: i) Fiber Length average (UHML), ii) Uniformity of length (UI), iii) Short fiber Index (SFI), iv) Fiber Strengh (STR), v) Elongation (ELG), vi) Micronaire (MIC), vii) Fiber Maturity (MR) (COSTA et al., 2006).

First, data were submitted to variance analysis (Test F) and Scott Knott test, both analyses carried out at significance level of 5\%. The phenotypic correlations (rf) among traits were evaluated at 1 and 5\% probability by $\mathrm{t}$-test with $\mathrm{n}-2$ degrees of freedom, where $\mathrm{n}$ corresponds to the number of genotypes evaluated.

Before performing the path analysis, multicollinearity diagnose and single multicollinearity degree of matrix $\mathrm{X}^{\prime} \mathrm{X}$ was established according to criteria suggested by Montgomery and Peck (1981), based on the condition number $(\mathrm{CN})$, which is the relation between the highest and lowest eigenvalue of matrix. If $\mathrm{CN}<100$, multicollinearity is weak and is not a problem for the analysis; if $100 \leq \mathrm{CN}<1000$, multicollinearity is considered moderate to strong; e if NC $\geq 1000$, is considered severe (CRUZ; REGAZZI; CARNEIRO, 2012). In addition, the phenotypic correlations were unfolded into direct and indirect effects of morphological traits and fiber quality (independent 
variables) on cotton seed yield (CSP) (dependent variable) through path analysis model proposed by Wright (1921; 1923). Statistical analyses and diagnosis of multicollinearity were performed by the software GENES (CRUZ, 2013).

\section{RESULTS AND DISCUSSION}

The variance analysis report of morphological and technological traits of fiber identified significant difference in three of eighteen traits evaluated, which evidences the genetic variability among genotypes evaluated.

Table 1. Mean squares (MS) and variance coefficients for 18 traits evaluated, in 21 cotton genotypes (Uberlândia, 2015).

\section{MEAN SQUARES}

\begin{tabular}{|c|c|c|c|c|c|c|c|c|c|c|c|c|c|c|c|c|c|c|c|}
\hline FV & $\begin{array}{l}\mathrm{G} \\
\mathrm{L}\end{array}$ & $\begin{array}{c}\text { TC } \\
\text { A }\end{array}$ & $\begin{array}{c}\text { TC } \\
\text { B }\end{array}$ & $\begin{array}{c}\mathrm{NF} \\
\mathrm{B}\end{array}$ & $\mathrm{NH}$ & FPB & SPB & $\begin{array}{l}\text { TP } \\
\text { B }\end{array}$ & LAI & CSP & LP & LY & $\begin{array}{l}\text { UH } \\
\text { ML }\end{array}$ & UI & SFI & $\begin{array}{c}\text { ST } \\
\text { R }\end{array}$ & $\begin{array}{c}\text { EL } \\
\text { G }\end{array}$ & $\begin{array}{l}\text { MI } \\
\text { C }\end{array}$ & $\begin{array}{l}\mathrm{M} \\
\mathrm{R}\end{array}$ \\
\hline $\begin{array}{c}\text { BLOC } \\
\text { OS }\end{array}$ & 2 & $\begin{array}{r}228 \\
.55 \\
\end{array}$ & $\begin{array}{r}92 . \\
74\end{array}$ & $\begin{array}{l}112 \\
.81\end{array}$ & 8.83 & $\begin{array}{c}150 . \\
04\end{array}$ & $\begin{array}{c}19.5 \\
6\end{array}$ & $\begin{array}{c}43.3 \\
2\end{array}$ & $\begin{array}{c}1800 \\
.19\end{array}$ & $\begin{array}{r}21177 \\
10.80\end{array}$ & $\begin{array}{c}25942 \\
4.93\end{array}$ & $\begin{array}{l}12 . \\
78\end{array}$ & $\begin{array}{c}4.6 \\
9\end{array}$ & $\begin{array}{c}6.1 \\
0\end{array}$ & $\begin{array}{c}4.8 \\
9\end{array}$ & $\begin{array}{c}0.5 \\
2\end{array}$ & $\begin{array}{c}0.2 \\
8\end{array}$ & $\begin{array}{c}0.2 \\
5\end{array}$ & $\begin{array}{c}0.0 \\
2\end{array}$ \\
\hline $\begin{array}{l}\text { GENÓ } \\
\text { TIPOS }\end{array}$ & $\begin{array}{l}2 \\
0 \\
\end{array}$ & $\begin{array}{l}3.5 \\
9 *\end{array}$ & $\begin{array}{l}1.4 \\
0^{\mathrm{ns}}\end{array}$ & $\begin{array}{l}1.1 \\
4^{\mathrm{ns}} \\
\end{array}$ & \begin{tabular}{|l}
12.1 \\
$0^{\text {ns }}$ \\
\end{tabular} & $\begin{array}{l}18.2 \\
8^{\text {ns }} \\
\end{array}$ & $\begin{array}{l}11.3 \\
3^{\mathrm{ns}} \\
\end{array}$ & $\begin{array}{l}13.3 \\
0^{\text {ns }} \\
\end{array}$ & $\begin{array}{l}127 . \\
73^{\text {ns }} \\
\end{array}$ & $\begin{array}{l}26504 \\
8.92^{\text {ns }} \\
\end{array}$ & $\begin{array}{l}51641 \\
.56^{\mathrm{ns}}\end{array}$ & $\begin{array}{l}5.2 \\
6 * \\
\end{array}$ & $\begin{array}{l}0.4 \\
0^{\mathrm{ns}}\end{array}$ & $\begin{array}{l}1.1 \\
3^{\text {ns }} \\
\end{array}$ & \begin{tabular}{|l}
0.4 \\
$4 \mathrm{~ns}$ \\
\end{tabular} & $\begin{array}{l}5.8 \\
0 * \\
\end{array}$ & $\begin{array}{l}0.2 \\
2^{\text {ns }}\end{array}$ & $\begin{array}{l}0.1 \\
3^{\text {ns }} \\
\end{array}$ & $\begin{array}{l}0.0 \\
0^{\mathrm{ns}}\end{array}$ \\
\hline $\begin{array}{c}\text { RESÍD } \\
\text { UO }\end{array}$ & $\begin{array}{l}4 \\
0 \\
\end{array}$ & $\begin{array}{c}1.7 \\
9 \\
\end{array}$ & $\begin{array}{c}1.1 \\
5 \\
\end{array}$ & $\begin{array}{c}1.5 \\
5 \\
\end{array}$ & $\begin{array}{c}13.1 \\
1 \\
\end{array}$ & $\begin{array}{c}25.2 \\
6 \\
\end{array}$ & $\begin{array}{c}15.1 \\
4 \\
\end{array}$ & $\begin{array}{c}12.1 \\
1 \\
\end{array}$ & $\begin{array}{c}325 . \\
24\end{array}$ & $\begin{array}{c}21998 \\
3.45 \\
\end{array}$ & $\begin{array}{c}40230 \\
.23 \\
\end{array}$ & $\begin{array}{c}2.6 \\
9 \\
\end{array}$ & $\begin{array}{c}0.5 \\
4 \\
\end{array}$ & $\begin{array}{c}0.9 \\
5 \\
\end{array}$ & $\begin{array}{c}0.5 \\
7 \\
\end{array}$ & $\begin{array}{c}1.5 \\
7 \\
\end{array}$ & $\begin{array}{c}0.1 \\
7 \\
\end{array}$ & $\begin{array}{c}0.1 \\
2 \\
\end{array}$ & $\begin{array}{c}0.0 \\
1 \\
\end{array}$ \\
\hline $\begin{array}{c}\text { TOTA } \\
\text { L }\end{array}$ & $\begin{array}{l}6 \\
2\end{array}$ & & & & & & & & & & & & & & & & & & \\
\hline \multicolumn{2}{|c|}{$\mathrm{CV}(\%)$} & $\begin{array}{c}3.3 \\
6\end{array}$ & $\begin{array}{c}6.9 \\
2\end{array}$ & $\begin{array}{c}10 . \\
8\end{array}$ & $\begin{array}{c}14.6 \\
4\end{array}$ & 8.51 & $\begin{array}{c}12.5 \\
7\end{array}$ & $\begin{array}{c}33.5 \\
4\end{array}$ & $\begin{array}{c}17.9 \\
7\end{array}$ & 46.14 & 55.74 & $\begin{array}{c}4.4 \\
1\end{array}$ & $\begin{array}{c}2.3 \\
2\end{array}$ & $\begin{array}{c}1.1 \\
4\end{array}$ & $\begin{array}{l}13 . \\
86\end{array}$ & $\begin{array}{c}3.9 \\
2\end{array}$ & $\begin{array}{c}6.4 \\
9\end{array}$ & $\begin{array}{c}7.9 \\
8\end{array}$ & $\begin{array}{c}1.1 \\
8\end{array}$ \\
\hline
\end{tabular}

(*) Probability significant at 5\%, according to F test; TCA = Chlorophyll content A; TCB = Chlorophyll content B; NFB = Number of fruitful branches; $\mathrm{NH}=1$ st fruit node height $(\mathrm{cm}) ; \mathrm{FPB}=$ First-position boll; $\mathrm{SPB}=$ Second-position boll; TPB = Third-position boll; LAI $=$ Leaf area index $(2 \mathrm{~cm}) ; \mathrm{CSP}=$ Cotton seed yield $\left(\mathrm{kg} . \mathrm{ha}^{-1}\right) ; \mathrm{LP}=$ Lint yield $\left(\mathrm{kg} . \mathrm{ha}^{-1}\right) ; \mathrm{LY}=$ Lint yield $(\%) ; \mathrm{UHML}=$ Fiber Length (mm); UI = Fiber Uniformity; SFI = Short fiber Index (\%); STR = Fiber strengh $\left(\mathrm{gf} \mathrm{tex}^{-1}\right)$; ELG = Ellongation (\%); MIC $=$ Micronaire Index and MR = Fiber Maturity

The experimental variation coefficient (CV) ranged from $1.14 \%$ for $55.74 \%$ IU for LP, but on average the values showed a good experimental precision. The CSP and LP presented the highest values of CV, but they are allowable because traits are governed by several genes, and, so, highly influenced by the environment. The experimental precision is of fundamental importance to achieve good results of genetic progress through selection in cotton breeding programs because reduces the expression of environmental effects on the genotypes (LEITE et al., 2015).

Table 2 showed averages grouping of 21 cotton genotypes for 3 traits evaluated. Chlorophyll content A (TCA), which is one of traits with significant effect and formed two groups, in which the genotypes: UFUJP15 - A (42.61), UFUJP15 - K (41.58), UFUJP15 - S (41.51), UFUJP15 - E (41.04) presented higher averages compared to commercial and other genotypes. Neves et al. (2005) reported that chlorophylls are pigments that convert light radiation into chemical energy, in form of ATP and NADPH, because of that, are closely related to the photosynthetic efficiency of plants, and so affecting growth, yield and adaptability to different environments.

Table 2. Averages grouping of 21 cotton genotypes according to Scott-knott method* evaluated from 3 traits (Uberlândia, 2015).

\begin{tabular}{|c|c|c|c|c|c|c|}
\hline Genotypes & TCA & & LY & & STR & \\
\hline DP 555 & 39,33 & $B$ & 40,88 & $\mathrm{a}$ & 33,53 & $\mathrm{a}$ \\
\hline FM 966 & 38,77 & $B$ & 39,32 & $b$ & 34,60 & $a$ \\
\hline UFU-A & 42,61 & $A$ & 37,24 & c & 32,07 & $b$ \\
\hline UFU-B & 38,64 & $B$ & 36,62 & $\mathrm{c}$ & 31,93 & $b$ \\
\hline UFU-C & 39,35 & $B$ & 36,38 & $\mathrm{c}$ & 35,30 & $a$ \\
\hline UFU-D & 40,10 & $B$ & 36,37 & c & 32,03 & $b$ \\
\hline UFU-E & 41,04 & $\mathrm{~A}$ & 36,18 & $\mathrm{c}$ & 30,43 & $b$ \\
\hline UFU-F & 39,97 & $\mathrm{~B}$ & 36,73 & c & 31,47 & $b$ \\
\hline UFU-G & 39,47 & $B$ & 37,48 & c & 31,37 & $b$ \\
\hline UFU-H & 38,78 & $B$ & 36,48 & $\mathrm{c}$ & 31,13 & $b$ \\
\hline UFU-J & 38,68 & $B$ & 36,73 & $\mathrm{c}$ & 29,90 & $b$ \\
\hline UFU-K & 41,58 & $\mathrm{~A}$ & 37,63 & $\mathrm{c}$ & 33,63 & $a$ \\
\hline UFU-L & 40,42 & $B$ & 35,45 & c & 31,33 & $b$ \\
\hline UFU-M & 39,41 & $B$ & 37,46 & c & 31,30 & $b$ \\
\hline UFU-N & 40,14 & $\mathrm{~B}$ & 37,97 & c & 31,90 & $b$ \\
\hline UFU-OB & 38,73 & $B$ & 36,20 & $\mathrm{c}$ & 31,90 & $\mathrm{~b}$ \\
\hline UFU-P & 38,67 & $B$ & 36,51 & $\mathrm{c}$ & 32,30 & $b$ \\
\hline UFU-R & 39,86 & $B$ & 37,98 & c & 30,73 & $b$ \\
\hline UFU-S & 41,51 & $A$ & 35,23 & c & 31,80 & $b$ \\
\hline UFU-T & 39,98 & $B$ & 38,97 & $b$ & 30,13 & $b$ \\
\hline UFU-Z & 39,63 & $B$ & 36,64 & c & 33,23 & $a$ \\
\hline
\end{tabular}


(*) Probability significant at 5\%. TCA $=$ Chlorophyll content A, LY $=$ Lint yield $(\%)$ and STR $=$ Fiber strengh $\left(\mathrm{gf} \mathrm{tex}^{-1}\right)$.

According to Fonseca; Santana (2002) fiber strength (STR) refers to specific breaking strength of a fibrous bundle from the fineness of the single fibers (tex) got from micronaire value, the researchers developed classification values: very low (less than 20), low (21-23), medium (24-26), high (27-29), very high (more than 30).

Three groups had been formed for lint yield. However, no genotype presented satisfactory result to the market ambitions, which recommend lint yield genotypes above 42\% (BORÉM, 2104). This is a result of climatic events because during cotton boll filling, there was water deficit and boll weevil attack, which interfered in adequate plant development and reduced the productive potential (ROSOLEM, 2014).

STR formed two groups, highlighting genotypes UFUJP15 - C (35.30 $\left.\mathrm{gf} \mathrm{tex}^{-1}\right)$, UFUJP15 - K (33.63 gf tex $\left.{ }^{-1}\right)$, UFUJP15 - Z (33.23 $\left.\mathrm{gf} \mathrm{tex}^{-1}\right)$ classified as having very high strength, and is considered by the textile industry with good fiber quality to wiring process.

Phenotypic correlations evaluation $\left(\mathrm{r}_{\mathrm{f}}\right)$. and interpretations, according to Nogueira et al. (2012) had to consider three aspects: magnitude, direction and significance. Positive correlations coefficients indicated predisposition to one variable increases when the other increases, and in negative correlations one variable increases while the other decreases.

This study chose phenotypic correlations for path analysis, because of differences between phenotypic and genotypic correlations. In accordance with Shukla et al. (1998) genotypic traits that do not correlate phenotypically may not have practical importance in selection, since it is often performed based on phenotype. Hoogerheide et al. (2007) also observed differences in the magnitudes of phenotypic and genotypic correlations, and thus, they chose phenotypic correlation for path analysis.

Table 3 showed traits with significant correlations in cotton seed yield: number of second-position boll (0.5054); lint yield (0.8853) and micronaire (0.5206). Hoogerheide et al. (2007) study of correlation between fiber technological traits and fiber yield in cotton genotypes, also found similar high correlations between CSP x MIC (0.77). Due to the positive correlation of CSP and MIC, when selecting genotypes of cotton with higher number of second-position bolls and fibers of higher micronaire values, indirectly, genotypes selected will have the highest cotton seed yield.

First and second-position bolls are responsible for the highest cotton seed yield, pursuant to the best lint yield, and also produce the highest quality fibers with high micronaire values.

The identification of traits with high correlation with major trait and highest direct effect favorable to selection, in such a way that the correlated response through the indirect selection is efficient, is very important for plants genetic breeding (CRUZ; REGAZZI; CARNEIRO, 2012). The yield is a trait of high complexity and dependent expression on other traits with direct or indirect influence (NOGUEIRA et al., 2012).

The highest significant positive phenotypic correlation was MIC x MR (0.9060) in others traits, which indicated to breeder that genotypes selection with higher micronaire value will indirectly be selecting the genotypes with fibers of highest maturity. This correlation is a result of micronaire that is an analysis of fiber fineness or diameter, and fiber maturity, which is the complete cellulose layers deposition that made the fiber. Thus, genotypes with highest diameters, that is, micronaire, has more space to a better distribution and deposition of cellulose layers that make up fully mature fibers.

The highest significant negative phenotypic correlation was FPB x TPB (- 0.7333). The negative correlation FPB x TPB (-0.6191) gives indications the genotypes selection with higher number of FPB, which are the bolls with the best fiber quality, will have lower number of SPB and even lower will be the number of thirdposition bolls. 
Table 3. Phenotypic correlations among characters evaluated in 21 cotton genotypes (Uberlândia, 2015).

\begin{tabular}{|c|c|c|c|c|c|c|c|c|c|c|c|c|c|c|c|c|c|c|}
\hline $\begin{array}{c}\text { TR } \\
\text { AIT }\end{array}$ & $\begin{array}{l}\mathrm{T} \\
\mathrm{C} \\
\mathrm{A}\end{array}$ & TCB & $\begin{array}{l}\mathrm{NF} \\
\mathrm{B}\end{array}$ & $\mathrm{NH}$ & FPB & SPB & TPB & LAI & CSP & LP & LY & $\begin{array}{l}\mathrm{UH} \\
\mathrm{ML}\end{array}$ & UI & SFI & $\begin{array}{c}\text { ST } \\
\text { R }\end{array}$ & ELG & MIC & MR \\
\hline $\begin{array}{c}\text { TC } \\
\text { A }\end{array}$ & 1 & $\begin{array}{l}0.57 \\
12 *\end{array}$ & $\begin{array}{l}0.2 \\
745\end{array}$ & $\begin{array}{c}0.13 \\
44\end{array}$ & $\begin{array}{c}0.00 \\
76\end{array}$ & $\begin{array}{c}0.12 \\
81\end{array}$ & $\begin{array}{c}0.02 \\
43\end{array}$ & $\begin{array}{c}0.07 \\
59\end{array}$ & $\begin{array}{c}0.20 \\
61\end{array}$ & $\begin{array}{c}0.27 \\
91\end{array}$ & $\begin{array}{c}- \\
0.14 \\
93 *\end{array}$ & $\begin{array}{c}0.11 \\
79\end{array}$ & $\begin{array}{c}0.35 \\
55\end{array}$ & $\begin{array}{c}0.32 \\
00\end{array}$ & $\begin{array}{c}0.05 \\
43\end{array}$ & $\begin{array}{c}0.15 \\
30\end{array}$ & $\begin{array}{c}0.38 \\
39\end{array}$ & $\begin{array}{c}- \\
0.38 \\
45\end{array}$ \\
\hline $\begin{array}{c}\text { TC } \\
\text { B }\end{array}$ & & 1 & $\begin{array}{l}0.0 \\
876\end{array}$ & $\begin{array}{c}0.09 \\
72\end{array}$ & $\begin{array}{c}- \\
0.11 \\
13\end{array}$ & $\begin{array}{c}- \\
0.11 \\
59\end{array}$ & $\begin{array}{c}0.35 \\
76\end{array}$ & $\begin{array}{c}0.06 \\
25\end{array}$ & $\begin{array}{c}0.03 \\
01\end{array}$ & $\begin{array}{c}0.21 \\
23\end{array}$ & $\begin{array}{c}- \\
0.13 \\
72\end{array}$ & $\begin{array}{l}0.08 \\
59 *\end{array}$ & $\begin{array}{c}- \\
0.20 \\
91\end{array}$ & $\begin{array}{c}0.10 \\
29\end{array}$ & $\begin{array}{c}0.00 \\
87\end{array}$ & $\begin{array}{c}- \\
0.23 \\
25\end{array}$ & $\begin{array}{c}0.51 \\
44\end{array}$ & $\begin{array}{c}- \\
0.43 \\
53\end{array}$ \\
\hline $\begin{array}{c}\mathbf{N F} \\
\mathbf{B}\end{array}$ & & & 1 & $\begin{array}{c}0.20 \\
03\end{array}$ & $\begin{array}{c}0.11 \\
34\end{array}$ & $\begin{array}{c}0.30 \\
52\end{array}$ & $\begin{array}{c}0.06 \\
13\end{array}$ & $\begin{array}{c}0.20 \\
55\end{array}$ & $\begin{array}{c}0.21 \\
50\end{array}$ & $\begin{array}{c}0.36 \\
17\end{array}$ & $\begin{array}{c}- \\
0.10 \\
12\end{array}$ & $\begin{array}{l}-\overline{-} \\
0.40 \\
49 *\end{array}$ & $\begin{array}{c}0.09 \\
29\end{array}$ & $\begin{array}{c}0.16 \\
26\end{array}$ & $\begin{array}{c}- \\
0.31 \\
31\end{array}$ & $\begin{array}{l}0.48 \\
03 *\end{array}$ & $\begin{array}{c}0.22 \\
62\end{array}$ & $\begin{array}{c}- \\
0.36 \\
99\end{array}$ \\
\hline NH & & & & 1 & $\begin{array}{c}0.00 \\
86\end{array}$ & $\begin{array}{c}0.21 \\
28\end{array}$ & $\begin{array}{c}0.05 \\
64\end{array}$ & $\begin{array}{c}0.27 \\
33\end{array}$ & $\begin{array}{c}0.35 \\
30\end{array}$ & $\begin{array}{c}0.26 \\
38\end{array}$ & $\begin{array}{c}0.12 \\
77\end{array}$ & $\begin{array}{c}0.07 \\
71\end{array}$ & $\begin{array}{c}0.04 \\
34\end{array}$ & $\begin{array}{c}0.12 \\
91\end{array}$ & $\begin{array}{c}0.10 \\
21\end{array}$ & $\begin{array}{c}0.19 \\
24\end{array}$ & $\begin{array}{c}0.00 \\
41\end{array}$ & $\begin{array}{c}0.11 \\
06\end{array}$ \\
\hline $\begin{array}{c}\text { FP } \\
\text { B }\end{array}$ & & & & & 1 & $\begin{array}{l}0.61 \\
91 *\end{array}$ & $\begin{array}{l}0.73 \\
33 *\end{array}$ & $\begin{array}{l}0.20 \\
06 *\end{array}$ & $\begin{array}{c}0.12 \\
55\end{array}$ & $\begin{array}{c}0.20 \\
99\end{array}$ & $\begin{array}{c}0.23 \\
01\end{array}$ & $\begin{array}{c}0.14 \\
64\end{array}$ & $\begin{array}{c}0.32 \\
71\end{array}$ & $\begin{array}{c}0.33 \\
87\end{array}$ & $\begin{array}{c}0.12 \\
01\end{array}$ & $\begin{array}{c}0.23 \\
93\end{array}$ & $\begin{array}{c}0.08 \\
77\end{array}$ & $\begin{array}{c}0.20 \\
45\end{array}$ \\
\hline$\overline{\text { SPB }}$ & & & & & & 1 & $\begin{array}{c}0.09 \\
06\end{array}$ & $\begin{array}{c}- \\
0.26 \\
46\end{array}$ & $\begin{array}{l}0.50 \\
54 *\end{array}$ & $\begin{array}{l}0.54 \\
90 *\end{array}$ & $\begin{array}{l}0.50 \\
79 *\end{array}$ & $\begin{array}{c}- \\
0.10 \\
23\end{array}$ & $\begin{array}{c}0.08 \\
91\end{array}$ & $\begin{array}{c}0.27 \\
31\end{array}$ & $\begin{array}{c}- \\
0.07 \\
00\end{array}$ & $\begin{array}{c}- \\
0.09 \\
88\end{array}$ & $\begin{array}{c}0.50 \\
78\end{array}$ & $\begin{array}{c}0.47 \\
47\end{array}$ \\
\hline $\begin{array}{l}\text { TP } \\
\text { B }\end{array}$ & & & & & & & 1 & $\begin{array}{c}- \\
0.10 \\
14\end{array}$ & $\begin{array}{c}-\overline{-} \\
0.04 \\
02\end{array}$ & $\begin{array}{c}0.03 \\
94\end{array}$ & $\begin{array}{c}0.00 \\
96\end{array}$ & $\begin{array}{c}0.19 \\
07\end{array}$ & $\begin{array}{l}0.48 \\
88 *\end{array}$ & $\begin{array}{c}0.39 \\
62\end{array}$ & $\begin{array}{c}-13 \\
0.13 \\
88\end{array}$ & $\begin{array}{c}- \\
0.30 \\
26\end{array}$ & $\begin{array}{c}- \\
0.13 \\
17\end{array}$ & $\begin{array}{c}0.01 \\
28\end{array}$ \\
\hline $\begin{array}{l}\text { LAI } \\
\end{array}$ & & & & & & & & 1 & $\begin{array}{c}0.24 \\
44\end{array}$ & $\begin{array}{c}0.16 \\
10\end{array}$ & $\begin{array}{c}0.03 \\
42\end{array}$ & $\begin{array}{c}0.01 \\
24\end{array}$ & $\begin{array}{c}0.28 \\
33\end{array}$ & $\begin{array}{c}0.18 \\
22\end{array}$ & $\begin{array}{c}0.42 \\
10\end{array}$ & $\begin{array}{c}0.11 \\
68\end{array}$ & $\begin{array}{c}0.21 \\
61\end{array}$ & $\begin{array}{c}0.28 \\
01\end{array}$ \\
\hline $\begin{array}{l}\text { CS } \\
\text { P }\end{array}$ & & & & & & & & & 1 & $\begin{array}{l}0.88 \\
53 *\end{array}$ & $\begin{array}{c}0.29 \\
47\end{array}$ & $\begin{array}{c}0.05 \\
56\end{array}$ & $\begin{array}{c}0.00 \\
45\end{array}$ & $\begin{array}{c}0.30 \\
69\end{array}$ & $\begin{array}{c}0.24 \\
35\end{array}$ & $\begin{array}{c}0.02 \\
25\end{array}$ & $\begin{array}{l}0.52 \\
06 *\end{array}$ & $\begin{array}{c}0.41 \\
25\end{array}$ \\
\hline $\mathbf{L P}$ & & & & & & & & & & 1 & $\begin{array}{c}0.38 \\
19\end{array}$ & $\begin{array}{c}0.08 \\
09\end{array}$ & $\begin{array}{c}- \\
0.04 \\
51\end{array}$ & $\begin{array}{c}0.23 \\
51\end{array}$ & $\begin{array}{c}0.23 \\
71\end{array}$ & $\begin{array}{c}\overline{-} \\
0.22 \\
12\end{array}$ & $\begin{array}{c}0.34 \\
26\end{array}$ & $\begin{array}{c}0.35 \\
85\end{array}$ \\
\hline$\overline{\mathbf{L Y}}$ & & & & & & & & & & & 1 & $\begin{array}{c}0.00 \\
00\end{array}$ & $\begin{array}{c}0.24 \\
44\end{array}$ & $\begin{array}{c}-\overline{0} \\
0.02 \\
14\end{array}$ & $\begin{array}{c}0.23 \\
57\end{array}$ & $\begin{array}{c}- \\
0.15 \\
70\end{array}$ & $\begin{array}{l}0.47 \\
41 *\end{array}$ & $\begin{array}{l}0.44 \\
89 *\end{array}$ \\
\hline $\begin{array}{l}\text { UH } \\
\text { ML }\end{array}$ & & & & & & & & & & & & 1 & $\begin{array}{c}0.35 \\
59\end{array}$ & $\begin{array}{l}0.67 \\
54 *\end{array}$ & $\begin{array}{c}0.24 \\
25\end{array}$ & $\begin{array}{c}0.46 \\
07\end{array}$ & $\begin{array}{c}0.15 \\
16\end{array}$ & $\begin{array}{c}0.03 \\
00\end{array}$ \\
\hline UI & & & & & & & & & & & & & 1 & $\begin{array}{l}0.63 \\
10^{*}\end{array}$ & $\begin{array}{c}0.21 \\
53\end{array}$ & $\begin{array}{c}0.01 \\
54\end{array}$ & $\begin{array}{l}0.23 \\
75 *\end{array}$ & $\begin{array}{l}0.10 \\
95 *\end{array}$ \\
\hline $\begin{array}{l}\text { SFI } \\
\end{array}$ & & & & & & & & & & & & & & 1 & $\begin{array}{c}0.27 \\
28\end{array}$ & $\begin{array}{c}0.02 \\
74\end{array}$ & $\begin{array}{c}0.14 \\
57\end{array}$ & $\begin{array}{c}0.07 \\
28\end{array}$ \\
\hline $\begin{array}{c}\text { ST } \\
\text { R }\end{array}$ & & & & & & & & & & & & & & & 1 & $\begin{array}{c}- \\
0.38 \\
46\end{array}$ & $\begin{array}{c}0.15 \\
40\end{array}$ & $\begin{array}{c}0.33 \\
88\end{array}$ \\
\hline $\begin{array}{c}\mathbf{E L} \\
\mathbf{G}\end{array}$ & & & & & & & & & & & & & & & & 1 & $\begin{array}{c}0.02 \\
60 \\
\end{array}$ & $\begin{array}{c}0.31 \\
53 \\
\end{array}$ \\
\hline $\begin{array}{c}\text { MI } \\
\text { C }\end{array}$ & & & & & & & & & & & & & & & & & 1 & $\begin{array}{l}0.90 \\
69 *\end{array}$ \\
\hline MR & & & & & & & & & & & & & & & & & & 1 \\
\hline $\begin{array}{c}\text { TR } \\
\text { AIT }\end{array}$ & $\begin{array}{l}\mathrm{T} \\
\mathrm{C} \\
\mathrm{A}\end{array}$ & TCB & $\begin{array}{c}\mathrm{NF} \\
\mathrm{B}\end{array}$ & $\mathrm{NH}$ & FPB & SPB & TPB & LAI & CSP & LP & LY & $\begin{array}{l}\mathrm{UH} \\
\mathrm{ML}\end{array}$ & UI & SFI & $\begin{array}{l}\text { ST } \\
\text { R }\end{array}$ & ELG & MIC & MR \\
\hline
\end{tabular}

(*) Probability significant at 5\%, according to F test; TCA = Chlorophyll content A; TCB = Chlorophyll content B; NFB = Number of fruitful branches; $\mathrm{NH}=1$ st fruit node height $(\mathrm{cm}) ; \mathrm{FPB}=$ First-position boll; SPB = Second-position boll; TPB = Third-position boll; LAI $=$ Leaf area index $(2 \mathrm{~cm}) ; \mathrm{CSP}=$ Cotton seed yield $\left(\mathrm{kg} . \mathrm{ha}^{-1}\right) ; \mathrm{LP}=$ Lint yield $\left(\mathrm{kg} . \mathrm{ha}^{-1}\right) ; \mathrm{FY}=$ Fiber yield $(\%) ; \mathrm{UHML}=$ Fiber Length $(\mathrm{mm})$; UI = Fiber Uniformity; SFI = Short fiber Index (\%); STR = Fiber strengh $\left(\mathrm{gf} \mathrm{tex}^{-1}\right)$; ELG = Ellongation (\%); MIC = Micronaire Index and MR = Fiber Maturity.

The significant positive correlations: SPB x CSP (0.5054); SPB x LP (0.5490); SPB x LY (0.5079) reported that select SPB will indirectly select genotypes with higher cotton seed yield, lint or fiber yield, which is very important agronomic trait. Therefore, the number of second-position bolls and first-position bolls are those that produce higher quantity of cotton seed and consequently more fiber. The negative correlations, involving the short fiber index (SFI), which is one of intrinsic trait of the fiber quality, SFI x UHML (-0.6754) and SFI x UI ($0.6310)$, indicated that the selection of fiber average length and uniformity will indirectly select genotypes with the lowest SFI, resulting in higher value-added fibers.

Although, Reddy et al. (2015) argue the study of correlation among traits is a useful association measurement, but does not allow the breeder gets conclusions about causes and effects of these associations. Silva et al. (2005) mention that path analysis provides a detailed understanding of traits influences. 
Before path analysis, multicollinearity diagnosis through condition number (CN) of phenotypic correlations matrices was carried out. The simultaneous inclusion of all the evaluated traits reported severe effects in the phenotypic correlation matrix. Thus, this study chose to eliminate NH, FPB, and STR that contributed to adverse effects of multicollinearity, and after, multicollinearity was also evaluated and had moderate to strong effect. Moreira et al. (2013) state that in order to circumvent multicollinearity adverse effects, traits that most contributed to this effect should be excluded.

Table 4 were not presented all traits because TCA, TCB, NRF, MTP, IAF, RF, UHML, UI, SFI, ELG and MR showed low correlations and their direct effects did not exceed the magnitude of the residual effect, so are not indicated for indirect selection. The effects of SPB, LP and MIC on cotton seed yield, SPB presented moderate positive correlation (0.5054) and low direct effect (0.0448), indicating that simultaneous selection for this trait does not provide a satisfactory gain in cotton seed yield. LP (0.8853) and MIC (0.5306) with positive correlations, high to moderate, respectively, and high direct effects, were: 0.6572 and 0.6285 respectively. Therefore, simultaneous selection with these traits presupposes satisfactory gain in cotton seed yield. Hoogerheide et al. (2007) found MIC with high positive correlation (0.77) and high direct effect (0.54), indicating truncated selection with this trait and may motivate a significant gain in cotton seed yield.

Table 4. Direct and indirect effects evaluation, which involved the main dependent trait of cotton seed yield and the independent ones: TCA, TCB, NFB, SPB, TPB, LAI, LP, LY, UHML, UI, SFI, ELG, MIC and MR in 21 cotton genotypes (Uberlândia, 2015).

\begin{tabular}{|c|c|c|}
\hline TRAIT & ASSOCIATION EFFECT & EVALUATION \\
\hline \multirow[t]{15}{*}{ SPB } & DIRECT BY CSP & 0.0448 \\
\hline & INDIRECT BY TCA & -0.0028 \\
\hline & INDIRECT BY TCB & -0.0102 \\
\hline & INDIRECT BY NFB & 0.0045 \\
\hline & INDIRECT BY TPB & 0.0003 \\
\hline & INDIRECT IBY LAI & -0.0704 \\
\hline & INDIRECT BY LP & 0.3608 \\
\hline & INDIRECT BY LY & -0.0595 \\
\hline & INDIRECT BY UHML & -0.0243 \\
\hline & INDIRECT BY UI & $\begin{array}{ll}-0.0071 \\
\end{array}$ \\
\hline & INDIRECT BY SFI & 0.0555 \\
\hline & INDIRECT BY ELG & -0.0211 \\
\hline & INDIRECT BY MIC & 0.3191 \\
\hline & INDIRECT BY MR & -0.0843 \\
\hline & TOTAL & 0.5054 \\
\hline \multirow[t]{15}{*}{ LP } & DIRECT BY CSP & 0.6572 \\
\hline & INDIRECT BY TCA & 0.0062 \\
\hline & INDIRECT BY TCB & 0.1870 \\
\hline & INDIRECT BY NFB & 0.0053 \\
\hline & INDIRECT BY SPB & 0.0246 \\
\hline & INDIRECT BY TPB & 0.0001 \\
\hline & INDIRECT BY LAI & 0.0429 \\
\hline & INDIRECT BY LY & -0.0447 \\
\hline & INDIRECT BY UHML & 0.0192 \\
\hline & INDIRECT BY UI & 0.0036 \\
\hline & INDIRECT BY SFI & 0.0478 \\
\hline & INDIRECT BY ELG & -0.0472 \\
\hline & INDIRECT BY MIC & 0.2153 \\
\hline & INDIRECT BY MR & -0.0636 \\
\hline & TOTAL & 0.8853 \\
\hline \multirow[t]{17}{*}{ MIC } & DIRECT BY CSP & 0.6285 \\
\hline & INDIRECT BY TCA & -0.0085 \\
\hline & INDIRECT BY TCB & -0.0453 \\
\hline & INDIRECT BY NFB & 0.0033 \\
\hline & INDIRECT BY SPB & 0.0227 \\
\hline & INDIRECT BY TPB & -0.0005 \\
\hline & INDIRECT BY LAI & -0.0575 \\
\hline & INDIRECT BY LP & 0.2252 \\
\hline & INDIRECT BY LY & -0.0555 \\
\hline & INDIRECT BY UHML & -0.0360 \\
\hline & INDIRECT BY UI & $\begin{array}{c}-0.0188 \\
\end{array}$ \\
\hline & INDIRECT BY SFI & 0.0296 \\
\hline & INDIRECT BY ELG & -0.0055 \\
\hline & INDIRECT BY MR & -0.1610 \\
\hline & TOTAL & 0.5206 \\
\hline & \multirow[t]{2}{*}{ EFFECT OF THE RESIDUAL VARIABLE } & 0.2171 \\
\hline & & 0.9529 \\
\hline
\end{tabular}

TCA = Chlorophyll content A; TCB = Chlorophyll content B; NFB = Number of fruitful branches; SPB = Second-position boll; $\mathrm{TPB}=$ Third-position boll; LAI $=$ Leaf area index $(2 \mathrm{~cm}) ; \mathrm{CSP}=$ Cotton seed yield $\left(\mathrm{kg} . \mathrm{ha}^{-1}\right) ; \mathrm{LP}=$ Lint yield $\left(\mathrm{kg} . \mathrm{ha}^{-1}\right) ; \mathrm{LY}=\mathrm{Lint}$ yield $(\%)$; UHML = Fiber Length $(\mathrm{mm})$; UI = Fiber Uniformity; SFI = Short fiber Index (\%); STR = Fiber strengh $\left(\mathrm{gf} \mathrm{tex}^{-1}\right) ; \mathrm{ELG}=$ Ellongation (\%); MIC = Micronaire Index and MR = Fiber Maturity. 
MIC and LP had a positive and relatively high effect among the traits studied in path analysis, indicating the presence of cause and effect. But just MIC is directly related to profits in cotton seed yield.

\section{CONCLUSIONS}

The intrinsic traits of fiber quality have influence on cotton seed yield, as well as, morphological traits. Indirect selection using SPB would not result in higher genotypes because it did not have a direct effect on cotton seed yield. Micronaire (MIC) for presenting positive correlation and direct effect on cotton seed yield can be used as an indirect selection criterion for cotton genotypes.

\section{REFERENCES}

Abrapa. Associação Brasileira De Produtores De Algodão. Estatísticas: O Algodão No Mundo. Disponível Em: Http://Www.Abrapa.Com.Br/Estatisticas/Paginas/Algodao-No-Brasil.Aspx. Acesso Em: 05 De Junho De $2016 \mathrm{a}$.

Abrapa. Associação Brasileira De Produtores De Algodão. Estatísticas: O Algodão No Brasil. Disponível Em: Http://Www.Abrapa.Com.Br/Estatisticas/Paginas/Algodao-No-Brasil.Aspx. Acesso Em: 02 De Junho De 2016 b.

Raújo, L. F.; Bertini, C. H. C.; Bleicher, E.; Vidal Neto, F. C.; Almeida, W. S. Características Fenológicas, Agronômicas E Tecnológicas Da Fibra Em Diferentes Cultivares De Algodoeiro Herbáceo. Revista Brasileira De Ciências Agrárias. Recife, V.8, N.3, P.448-453, Mar. 2013.

Azevedo, A. M.; Seus, R.; Gomes, C. L.; Freitas, E. M.; Candido, D. M.; Silva, D. J. H.; Carneiro, P. C. S. Correlações Genotípicas E Análise De Trilha Em Famílias De Meios-Irmãos De Couve De Folhas. Pesquisa Agropecuária Brasileira. Brasília, V.51, N.1, P. 35-44, Jan. 2016.

Bélot, J. L.; Marquié, C. Novos Usos Podem Aumentar Importância Do Caroço. In: Revista Visão Agrícola, V. 6, P.112-114, 2006.

Cardoso, D. B.O.; Mundim, F. M.; Sousa, L. B. Variabilidade Genética E Coeficiente De Determinação Em Genótipos De Algodoeiro Quanto A Qualidade Da Fibra. Revista Verde. Pombal, V. 10, N. 3, P 66 - 71, Jul.- Set. 2015.

Conab. Companhia Nacional De Abastecimento. Acompanhamento Da Safra Brasileira De Grãos, V. 3 - Safra 2015/2016, N.7 - Sétimo Levantamento, Abr. 2016.

Conab. Perspectivas Para A Agropecuária / Companhia Nacional De Abastecimento - V.2 - Brasília: Conab, 2014 - V.1. Anual. Disponível Em: Http://Www.Conab.Gov.Br.. Acesso Em 27 Out. 2015.

Costa, J. N.; Santana, J. C. F.; Wanderley, M. J. R.; Sobrinho, R. E. V. Padrões Universais Para Classificação De Algodão. Embrapa Algodão: Documentos 151. Campina Grande, 22p. 2006.

Coutinho, C. R.; Andrade, J. A. S.; Pegoraro, R. F. Produtividade E Qualidade De Fibra De Cultivares De Algodoeiro (Gossypium Hirsutum L.) Na Região Do Semiárido Mineiro. Revista Essentia. Sobral, V. 16, N. 2, P. 62-68, Jan/Jun. 2015.

Cruz, C.D. Genes - A Software Package For Analysis In Experimental Statistics And Quantitative Genetics. Acta Scientiarum. V.35, N.3, P.271-276, 2013

Cruz, C.D.; Regazzi, A.J.; Carneiro, P.C.S. Modelos Biométricos Aplicados Ao Melhoramento Genético. Viçosa, Mg: Ufv, 2012. V. 1, 514 P.

Entringer, G. C.; Santos, P. H. A. D.; Vettorazzi, J. C. F.; Cunha, K. S.; Pereira, M. G. Correlação E Análise De Trilha Para Componentes De Produção De Milho Superdoce. Revista Ceres. Viçosa, V. 61, N. 3, P. 356-361, Mai./Jun., 2014.

Fideles Filho, J.; Beltrão, N. E.M; Pereira, A. S. Desenvolvimento De Uma Régua Para Medidas De Área Foliar Do Algodoeiro. Revista Brasileira De Engenharia Agrícola E Ambiental, V. 14, N. 7, P 736-741, 2010.

Fonseca, R. G.; Santana, J. C. F. Resultados De Ensaio Hvi E Suas Interpretações (Astm D-4605). Embrapa. Campina Grande. Dez. 2002. 13p (Circular Técnica, 66).

Hoogerheide, E. S. S.; Vencovshy, R.; Farias, F. J. C.; Freire, E. C.; Arantes, E. M. Correlações E Análise De Trilha De Caracteres Tecnológicos E A Produtividade De Fibra De Algodão. Pesquisa Agropecuária Brasileira. Brasília, V.42, N.10, P. 1401-1405, Out. 2007.

Leite, W. S.; Pavan, B. E.; Matos Filho, C. H. A.; Feitosa, F. S.; Oliveira, C. B. Estimativas De Parâmetros Genéticos E Correlações Entre Caracteres Agronômicos Em Genótipos De Soja. Revista Nativa. Sinop, V. 03, N. 04, P. 241-245, Out./Dez. 2015.

Lúcio, A. D.; Storck, L.; Krause, W.; Gonçalves, R. Q.; Nied, A. H. Relações Entre Os Caracteres De Maracujazeiro-Azedo. Revista Ciência Rural. Santa Maria, V. 43, N. 2, P. 225-232, Fev. 2013.

Genetics and Molecular Research 16 (3): gmr16039805 
Mapa. Ministério Da Agricultura, Pecuária E Abastecimento. Projeções Do Agronegócio. Brasil 2014/15 A 2024/25 -Projeções De Longo Prazo. Brasília, Jul. 2015.

Montgomery, D. C.; Peck E. A. Introduction To Linear Regression Analysis. New York: John Wiley E Sons 1981. 504p.

Moreira, S. O.; Gonçalves, L. S. A.; Rodrigues, R.; Sudré, C. P.; Amaral Júnior, A. T.; Medeiros, A. M. Correlações E Análise De Trilha Sob Multicolinearidade Em Linhas Recombinadas De Pimenta (Capsicum Annuum L.). Revista Brasileira De Ciências Agrárias. Recife, V.8, N.1, P. 15-20, 2013.

Nogueira, A. P. O.; Sediyama, T.; Sousa, L. B.; Hamawaki, O. T.; Cruz, C.D.; Pereira, D.G.; Matsuo, E. Análise De Trilha E Correlações Entre Caracteres Em Soja Cultivada Em Duas Épocas De Semeadura. Bioscience Journal, Uberlândia, V. 28, N. 6, P. 877-888, Nov./Dec. 2012.

Ramalho, M.A.P.; Et Al. Aplicações Da Genética Quantitativa No Melhoramento De Plantas Autógamas. Ed. Lavras: Ed. Ufla, Lavras, 2012, 522p.

Reddy, K. B.; Reddy, V. C.; Ahmed, M. L.; Naidu, T. C. M.; Srinivasarao, V. Correlation And Path Coefficient Analysis In Upland Cotton (Gossypium Hirsutum L.). International Journal Of Pure \& Applied Bioscience, V. 3, N. 3, P. 70-80, 2015.

Rios, S. A.; Borém, A.; Guimarães, P. E. O.; Paes, M. C. D. Análise De Trilha Para Carotenoides Em Milho. Revista Ceres. Viçosa, V. 59, N.3, P. 368-373, Mai./Jun. 2012.

Salla, V. P.; Danner, M. A.; Citadin, I.; Sasso, S. A. Z.; Donazzolo, J.; Gil, B. V. Análise De Trilha Em Caracteres De Frutos De Jabuticabeira. Pesquisa Agropecuária Brasileira. Brasília, V.50, N. 3, P. 218-223, Mar. 2015.

Shukla, S.; Singh, K.; Pushpendra. Correlation And Path Coefficient Analysis Of Yield And Its Components In Soybean (Glycine Max (L.) Merrill). Soybean Genetics Newsletter, V.25, P.67-70,1998.

Silva, A. A.; Pires, M. M. Os Impactos Da Proalba Na Cotonicultura Do Estado Da Bahia. Revista Brasileira De Gestão E Desenvolvimento Regional. Taubaté, V.11, N. 3, P. 4-30, Set.-Dez. 2015.

Silva, S. A.; Carvalho, F. I. F.; Nedel, J. L.; Cruz, P. J.; Silva, J. A. G.; Caetano, V. R.; Hartwing, I.; Sousa, C. S. Análise De Trilha Para Os Componentes De Rendimento De Grãos Em Trigo. Bragantia, Campinas, V. 64, N. 2, P. 191-196, 2005.

Teixeira, D. H. L.; Oliveira, M. S. P.; Gonçalves, F. M. A.; Nunes, J. A. R. Correlações Genéticas E Análise De Trilha Para Componentes Da Produção De Frutos De Açaizaeiro. Revista Brasileira De Fruticultura. Jaboticabal - Sp, V. 34, N. 4, P. 1135-1142, Dez. 2012.

Toebe, M.; Cargnelutti Filho, A. Não Normalidade Multivariada E Multicolinearidade Na Análise De Trilha Em Milho. Pesquisa Agropecuária Brasileira. Brasília, V. 48, N. 5, P. 466-477, Maio. 2013.

Wright, S. Correlation And Causation. Journal Of Agricultural Research, V.20, P.557-585, 1921. Disponível Em: <Http://Www.Biodiversitylibrary.Org/Item/36231\#Page/756/Mode/1up>. Acesso Em: 05 Jul. 2016.

Wright, S. The Theory Of Path Coefficients - A Replay To Niles' Criticismo. Genetics, V.52, P.239-255, 1923. Disponível Em: Http://Www.Genetics.Org/Content/Genetics/8/3/239.Full.Pdf. Acesso Em: 05 Jul. 2016. 